\title{
Quantum transport in chains with noisy off-diagonal couplings
}

\author{
Andrey Pereverzev and Eric R. Bittner \\ Department of Chemistry and Center for Materials Chemistry, \\ University of Houston \\ Houston, TX 77204
}

(Dated: May 10, 2018)

\begin{abstract}
We present a model for conductivity and energy diffusion in a linear chain described by a quadratic Hamiltonian with Gaussian noise. We show that when the correlation matrix is diagonal, the noiseaveraged Liouville-von Neumann equation governing the time-evolution of the system reduces to the Lindblad equation with Hermitian Lindblad operators. We show that the noise-averaged density matrix for the system expectation values of the energy density and the number density satisfy discrete versions of the heat and diffusion equations. Transport coefficients are given in terms of model Hamiltonian parameters. We discuss conditions on the Hamiltonian under which the noiseaveraged expectation value of the total energy remains constant. For chains placed between two heat reservoirs, the gradient of the energy density along the chain is linear.
\end{abstract}

\section{INTRODUCTION}

The problem of energy and charge transport in lowdimensional systems has attracted considerable current attention. 1, 2, 3, 4, [5, 6]. This recent interest is primarily driven by new advances in nanotechnology and the resulting need to explain new and unusual transport properties of nanomaterials [7, [8]. In many respects, such materials behave as effectively one or two dimensional systems. Furthermore, from a theoretical standpoint, this tnterest is driven by the desire to understand how dimensionality affects transport properties and whether normal transport is possible in low dimensions, and, if so, what are the conditions for such behavior.

Studies of heat transport in one dimensional models have long and rich history. Review articles on heat transport in one dimension include Refs. [9] and [10]. The earliest studies addressing this problem for anharmonic chains include the seminal numerical simulations by Fermi, Pasta and Ulam [1] and Peierls' discussion of the constraints on phonon interactions in one dimension 12. More recent theoretical studies of heat transport in one dimension include Refs. 10, 13, 14, 15, 16]. Extensive numerical simulations have also been reported in recent years [10, 17, 18, 19, 20]. It is now well established that thermal transport in momentum conserving one dimensional models is anomalous, i.e. the Fourier heat law is not observed in this systems. The physical reason for such behavior is in the slow decay of normal modes with small wave numbers. In one dimension, the fast propagation of heat carried by these modes makes normal heat transport impossible.

Due to the difficulties in the theoretical treatment of one-dimensional transport, exactly solvable models are of interest. One of the few one dimensional models that shows normal heat conductivity is a stochastic and nonHamiltonian model introduced by Bolsterli et al. 21, 22]. In this paper we consider a quantum mechanical Hamiltonian model in which the Hamiltonian matrix elements are taken as time dependent stochastic variables. We show that upon averaging over the noise, one obtains a
Lindblad-type equation for the evolution of the quantum density matrix. We then go on to give the conditions upon the Hamiltonian which produce normal (i.e. diffusive) energy and particle transport. Finally, we demonstrate the validity of our model using numerical simulations.

\section{GAUSSIAN WHITE NOISE HAMILTONIANS}

The Hamiltonian of the one-dimensional model that we will consider in this paper belongs to a general class of Hamiltonians with Gaussian white noise

$$
H=H_{0}+\sum_{i} \lambda_{i}(t) V_{i}
$$

where $H_{0}$ and $V_{i}$ are arbitrary Hermitian operators and gaussian stochastic coefficients $\lambda_{i}(t)$ have average mean $\overline{\lambda_{i}(t)}=0$ and second moments given by

$$
\overline{\lambda_{i}(t) \lambda_{j}\left(t^{\prime}\right)}=g_{i j} \delta\left(t-t^{\prime}\right) .
$$

Hamiltonians such as this can model a wide variety of physical situations where the motion or transport is driven by an external field. One such example is the case of Förster resonant excitation transfer in biomolecules where the migration and diffusion of an initial electronic excitation within the system is dependent upon the local environmental and conformational fluctuations that modulate the off-diagonal couplings.

From a theoretical standpoint, such Hamiltonians are useful since one can explicitly average over the noise when calculating the time evolution of the density matrix or expectation values of various observables. Noiseaveraged time evolution for various specific forms of the Hamiltonian in Eq. (11) have been considered previously [23, 24, 25, 26, 27]. We give here a brief discussion of noise-averaged time evolution for the general case and then go on to emphasize points that are relevant for the studies of heat transport. 
In general, the quantum density matrix satisfies the Liouville-von Neumann equation,

$$
i \frac{\partial \rho}{\partial t}=\left(\mathcal{L}_{0}+\mathcal{L}_{V}(t)\right) \rho
$$

Action of the superoperators $\mathcal{L}_{0}$ and $\mathcal{L}_{V}$ on $\rho$ is given, respectively, by $\mathcal{L}_{0} \rho=\frac{1}{\hbar}\left[H_{0}, \rho\right]$ and $\mathcal{L}_{V}(t) \rho=$ $\frac{1}{\hbar} \sum_{i} \lambda_{i}(t)\left[V_{i}, \rho\right]$. The density matrix at time $t$ is given in terms of the density matrix $\rho(0)$ at time $t=0$ by

$$
\rho(t)=\mathcal{U}(t) \rho(0)
$$

Here the time-evolution superoperator $\mathcal{U}(t)$ is given by the following infinite series:

$$
\mathcal{U}(t)=e^{-i \mathcal{L}_{0} t}-i \int_{0}^{t} d \tau e^{-i \mathcal{L}_{0}(t-\tau)} \mathcal{L}_{V}(\tau) e^{-i \mathcal{L}_{0} \tau}-\int_{0}^{t} d \tau \int_{0}^{\tau} d \tau^{\prime} e^{-i \mathcal{L}_{0}(t-\tau)} \mathcal{L}_{V}(\tau) e^{-i \mathcal{L}_{0}\left(\tau-\tau^{\prime}\right)} \mathcal{L}_{V}\left(\tau^{\prime}\right) e^{-i \mathcal{L}_{0} \tau^{\prime}}+\ldots
$$

Noise-averaged expectation values of an operator $O$ are computed using

$$
\overline{\langle O(t)\rangle}=\operatorname{Tr}(\overline{O(t) \mathcal{U}(t) \rho(0)}) .
$$

It is assumed here that operator $O$ can have an explicit time dependence. When performing averages as in Eq. (6), we need to distinguish between two types of operators: those with and those without stochastic coefficients $\lambda_{i}(t)$. In the latter case, averaging over noise as in Eq. 6] reduces to an averaging of the evolution superoperator $\mathcal{U}(t)$ and such expectation values can be calculated with the noise averaged density matrix.

Noise averaging of $\mathcal{U}(t)$ can be performed by taking averages for each term in the series and then resumming the series. This involves averaging products of the stochastic coefficients. Since $\lambda_{i}(t)$ is sampled from a Gaussian deviate, all terms involving an odd number of coefficients necessarily vanish. Furthermore, any term with an even number of terms can be written as a sum of all possible products of second moments. However, due to the order of integrations over time in Eq. (5) and the fact that second moments in Eqs. (2) involve delta functions in time, only one product from the sum contributes to the average after all the time integrations are performed. For example, the fourth order average $\overline{\lambda_{i}(t) \lambda_{j}(\tau) \lambda_{k}\left(\tau^{\prime}\right) \lambda_{l}\left(\tau^{\prime \prime}\right)}$, decomposes into the following second-order terms:

$$
\begin{aligned}
& \overline{\lambda_{i}(t) \lambda_{j}(\tau) \lambda_{k}\left(\tau^{\prime}\right) \lambda_{l}\left(\tau^{\prime \prime}\right)}=g_{i j} g_{k l} \delta(t-\tau) \delta\left(\tau^{\prime}-\tau^{\prime \prime}\right) \\
& +g_{i k} g_{j l} \delta\left(t-\tau^{\prime}\right) \delta\left(\tau-\tau^{\prime \prime}\right)+g_{i l} g_{j k} \delta\left(t-\tau^{\prime \prime}\right) \delta\left(\tau-\tau^{\prime}\right)
\end{aligned}
$$

Since the region of integration for the fourth order term in the series (5) is $t \geq \tau \geq \tau^{\prime} \geq \tau^{\prime \prime}$ we can see that only the $g_{i j} g_{k l} \delta(t-\tau) \delta\left(\tau^{\prime}-\overline{\tau^{\prime \prime}}\right)$ term will contribute to the series. Similar analysis can be applied to all even order terms.

Averaging over noise and resumming the series given by Eq. (15) produces

$$
\overline{\mathcal{U}(t)}=e^{-i \mathcal{L}_{0} t-\mathcal{M} t}
$$

where the action of the superoperator $\mathcal{M}$ on the density matrix $\rho$ is given by

$$
\mathcal{M} \rho=\frac{1}{2 \hbar^{2}} \sum_{i j} g_{i j}\left[V_{i},\left[V_{j}, \rho\right]\right] .
$$

Hence, our noise-averaged propagation is unitary and norm-conserving. It follows from Eq. (8) that the noiseaveraged density matrix satisfies the following equation

$$
i \frac{\partial \bar{\rho}}{\partial t}=\left(\mathcal{L}_{0}-i \mathcal{M}\right) \bar{\rho}
$$

This allows one to see an interesting connection between noise-averaged time evolution of the density matrix for the noisy system and the time evolution of the reduced density matrix for an open quantum system.

Consider the case when correlation matrix $g_{i j}$ in Eq. (9) is diagonal, i.e. $g_{i j}=g_{i i} \delta_{i j}$. In this case, Eq. (10) can be rewritten as

$$
i \frac{\partial \bar{\rho}}{\partial t}=\frac{1}{\hbar}\left[H_{0}, \bar{\rho}\right]-\frac{i}{2 \hbar^{2}} \sum_{i} g_{i i}\left[V_{i},\left[V_{i}, \bar{\rho}\right]\right] .
$$

This has the form of a Lindblad equation with Hermitian Lindblad operators 28]. Thus, the time evolution of the reduced density matrix under the Lindblad equation with Hermitian operators is equivalent to noise-averaged unitary evolution with Hamiltonian (1) in the special case of a diagonal correlation matrix $g_{i j}$. Connection between Lindblad-type evolution with Hermitian Lindblad operators and an averaged random unitary evolution was discussed in Ref. 29]. It is interesting that the unitary operator proposed in Ref. [29] is different from the unitary operator corresponding to the Hamiltonian in Eq. (1). This implies that averaging different unitary evolutions over the same noise spectrum can ultimately lead to the same Lindblad-type evolution.

If the operator whose noise-averaged expectation value we want to find depends on the coefficients $\lambda_{i}(t)$ then these coefficients must be taken into account when performing averages as in Eq. 6] Let us consider the linear case $\lambda_{i}(t) W$ where $W$ is time independent and $\lambda_{i}(t)$ is a 
gaussian random variable. An example of an operator of this type is the second term of Eq. 11 Using series (5) for superoperator $\mathcal{U}(t)$ and substituting $\lambda_{i}(t) W$ for $O$ in
Eq.(6) we can again perform averages for each term and resum the series to obtain

$$
\operatorname{Tr}\left(\overline{\lambda_{i}(t) W \mathcal{U}(t) \rho(0)}\right)=-\frac{i}{\hbar} \operatorname{Tr}\left(W \sum_{j} g_{i j}\left[V_{j}, e^{-i \mathcal{L}_{0} t-\mathcal{M} t} \rho(0)\right]\right)=-\frac{i}{\hbar} \operatorname{Tr}\left(\overline{\rho(t)} \sum_{j} g_{i j}\left[W, V_{j}\right]\right)
$$

where the last line is obtained by using cyclic permutations under the trace and the fact that $e^{-i \mathcal{L}_{0} t-\mathcal{M} t} \rho(0)=\overline{\rho(t)}$.

Explicit averaging over noise was performed in Eqs. (8) and (12). However, these expressions remain mostly formal. For some forms of operators $H_{0}$ and $V_{i}$ further exact treatment is possible. One such example is $H_{0}$ and $V_{i}$ that are quadratic forms of creations and annihilation operators. Hamiltonians of this type were used in solid state physics to describe electronic transport under the influence of an external driving field such as lattice vibrations [26, 30]. These models gave a satisfactory picture of electron diffusion. However, such models generally do not conserve average energy of the system and therefore cannot show normal heat propagation.

Let us use Eqs. (8) and (12) to determine the precise conditions that the operators $H_{0}$ and $V_{i}$ must satisfy in order for the noise-averaged expectation value of the total energy to remain constant. The noise-averaged energy expectation value is given by

$$
\overline{\langle H(t)\rangle}=\overline{\left\langle H_{0}(t)\right\rangle}+\sum_{i} \overline{\lambda_{i}(t)\left\langle V_{i}(t)\right\rangle}
$$

Since $H_{0}$ does not depend upon the stochastic coefficients, we obtain

$$
\overline{\left\langle H_{0}(t)\right\rangle}=\operatorname{Tr}\left(H_{0} e^{-i \mathcal{L}_{0} t-\mathcal{M} t} \rho(0)\right) .
$$

To find the second term on the right hand side of Eq. (13) we use Eq. (12) where we need to put $V_{i}$ for $W$ and sum over all $i$. We obtain

$$
\sum_{i} \overline{\lambda_{i}(t)\left\langle V_{i}(t)\right\rangle}=-\frac{i}{\hbar} \operatorname{Tr}\left(\overline{\rho(t)} \sum_{i j} g_{i j}\left[V_{i}, V_{j}\right]\right)
$$

Since matrix $g_{i j}$ is always symmetric, the last quantity vanishes for arbitrary $V_{i}$ 's and, therefore, does not contribute to $\overline{\langle H(t)\rangle}$. Thus we have

$$
\overline{\langle H(t)\rangle}=\overline{\left\langle H_{0}(t)\right\rangle}
$$

For $\overline{\langle H(t)\rangle}$ to be constant in time, its time derivative must vanish. Differentiating $\overline{\left\langle H_{0}(t)\right\rangle}$ with respect to time we obtain

$$
\frac{d \overline{\left\langle H_{0}(t)\right\rangle}}{d t}=\operatorname{Tr}\left(H_{0}\left(-i \mathcal{L}_{0}-\mathcal{M}\right) \overline{\rho(t)}\right) .
$$

Using the fact that the trace is invariant under cyclic permutations, the symmetric nature of matrix $g_{i j}$, and that $\mathcal{L}_{0} H_{0}=0$, we obtain

$$
\frac{d \overline{\left\langle H_{0}(t)\right\rangle}}{d t}=-\operatorname{Tr}\left(\overline{\rho(t)} \mathcal{M} H_{0}\right) .
$$

For arbitrary $\rho(0)$ this derivative will be equal to zero if

$$
\mathcal{M} H_{0}=\frac{1}{2 \hbar^{2}} \sum_{i j} g_{i j}\left[V_{i},\left[V_{j}, H_{0}\right]\right]=0 .
$$

This is the condition for $\overline{\langle H(t)\rangle}$ to be constant in time. Examples of operators $H_{0}$ and $V_{i}$ for which Eq. (19) is true include the case when $H_{0}$ and $V_{i}$ satisfy the condition $\left[H_{0}, V_{i}\right]=$ const for all $i$, in particular when $H_{0}$ and $V_{i}$ commute.

\section{MODEL HAMILTONIAN AND OBSERVABLES}

We now apply the results of the previous section to study quantum heat conduction and diffusion in a onedimensional chain of $N$ sites described by the following Hamiltonian

$$
\begin{aligned}
H= & \hbar \omega \sum_{i}^{N} a_{i}^{\dagger} a_{i}+\hbar \sum_{i=1}^{N} \mu_{i}(t)\left(a_{i}^{\dagger} a_{i+1}+a_{i+1}^{\dagger} a_{i}\right) \\
& +i \hbar \sum_{i=1}^{N} \nu_{i}(t)\left(a_{i}^{\dagger} a_{i+1}-a_{i+1}^{\dagger} a_{i}\right)
\end{aligned}
$$

Here $a_{i}^{\dagger}$ and $a_{i}$ denote, respectively, creation and annihilation operators for quasi-particles at chain site $i$. These quasi-particles can be bosons, fermions, or spin excitations. The stochastic hopping coefficients $\mu_{i}(t)$ and $\nu_{i}(t)$ are realizations of the Gaussian noises with zero mean. We choose the following simple expressions for the second moments,

$$
\begin{aligned}
\overline{\mu_{j}(t) \mu_{k}\left(t^{\prime}\right)} & =\alpha_{1}^{2} \delta_{j k} \delta\left(t-t^{\prime}\right), \\
\overline{\nu_{j}(t) \nu_{k}\left(t^{\prime}\right)} & =\alpha_{2}^{2} \delta_{j k} \delta\left(t-t^{\prime}\right) \\
\overline{\mu_{j}(t) \nu_{k}\left(t^{\prime}\right)} & =0
\end{aligned}
$$


We will consider two types of boundary conditions: cyclic and open ends. For the former we have $a_{N+1}=a_{1}$, $a_{N+1}^{\dagger}=a_{1}^{\dagger}$, and for the latter $a_{N+1}=a_{N+1}^{\dagger}=0$.

The model Hamiltonian in Eq. (20) belongs to the class of noisy Hamiltonians we just described. Indeed, we can rewrite Eq. [20] as

$$
H=H_{0}+\sum_{j=1}^{2 N} \lambda_{j}(t) V_{j}
$$

where

$$
H_{0}=\hbar \omega \sum_{i}^{N} a_{i}^{\dagger} a_{i}
$$

and

$$
\lambda_{j}(t) V_{j}=\hbar \mu_{i}(t)\left(a_{i}^{\dagger} a_{i+1}+a_{i+1}^{\dagger} a_{i}\right)
$$

for even $j$ 's and

$$
\lambda_{j}(t) V_{j}=i \hbar \nu_{i}(t)\left(a_{i}^{\dagger} a_{i+1}-a_{i+1}^{\dagger} a_{i}\right)
$$

for odd j's. Thus, discussion of Sec. II can be applied to the studies of time evolution driven by this Hamiltonian. Note that Hamiltonian (20) satisfies the average energy constraint in Eq. (19) because its time independent part $H_{0}$ commutes with all $V_{i}$ 's. Therefore noise averged expectation value of the total energy remains constant in time and we can discuss heat conduction in this model.

Heat conduction and particle diffusion can be studied by considering time evolution of expectation values of energy density and number density averaged over noise. For both types of boundary conditions, operators for the number density $n_{i}$ are

$$
n_{i}=a_{i}^{\dagger} a_{i}
$$

The energy density $\epsilon_{i}$ at site $i$ for cyclic boundary conditions is given

$$
\begin{aligned}
\epsilon_{i}= & \hbar \omega a_{i}^{\dagger} a_{i}+\frac{1}{2} \hbar \mu_{i}(t) A_{i}+\frac{1}{2} \hbar \mu_{i-1}(t) A_{i-1} \\
& +\frac{1}{2} \hbar \nu_{i}(t) B_{i}+\frac{1}{2} \hbar \nu_{i-1}(t) B_{i-1}
\end{aligned}
$$

where $A_{i}=\left(a_{i}^{\dagger} a_{i+1}+a_{i+1}^{\dagger} a_{i}\right)$ and $B_{i}=i\left(a_{i}^{\dagger} a_{i+1}-a_{i+1}^{\dagger} a_{i}\right)$ In the case of open-end boundaries, operators for the energy density at site $i$ are the same as for cyclic boundaries except for the first and last sites where we use

$$
\begin{aligned}
\epsilon_{1} & =\hbar \omega a_{1}^{\dagger} a_{1}+\frac{1}{2} \hbar \mu_{1}(t) A_{1}+\frac{1}{2} \hbar \nu_{1}(t) B_{1} \\
\epsilon_{N} & =\hbar \omega a_{N}^{\dagger} a_{N}+\frac{1}{2} \hbar \mu_{N-1}(t) A_{N-1}+\frac{1}{2} \hbar \nu_{N-1}(t) B_{N-1}
\end{aligned}
$$

Note that the number density operator is independent of the noise. The energy density, however, has both a noise independent component (which is proportional to number density) and a noisy component linear in coefficients $\mu_{i}(t)$ or $\nu_{i}(t)$. Thus, we will need to take both types of averages over noise discussed in Sec. II] We have for $\overline{\left\langle n_{i}(t)\right\rangle}$,

$$
\overline{\left\langle n_{i}(t)\right\rangle}=\operatorname{Tr}\left(n_{i} e^{-i \mathcal{L}_{0} t-\mathcal{M} t} \rho(0)\right) .
$$

Following procedure in Sec. II and using the explicit form of the second moments (Eq. 21) we obtain,

$$
M \rho=\frac{\alpha_{1}^{2}}{2} \sum_{i}\left[A_{i},\left[A_{i}, \rho\right]\right]+\frac{\alpha_{2}^{2}}{2} \sum_{i}\left[B_{i},\left[B_{i}, \rho\right]\right] .
$$

Here the summations extend from 1 to $N$ for cyclic boundaries and from 1 to $N-1$ for open-end boundaries. In the case of $\overline{\left\langle\epsilon_{i}(t)\right\rangle}$, we can evaluate the noise-dependent part using Eq. 12 Substituting $A_{i}$ or $B_{i}$ for $W$ and using Eq. 21 we obtain

$$
\overline{\left\langle\mu_{i}(t) A_{i}(t)\right\rangle}=\overline{\left\langle\nu_{i}(t) B_{i}(t)\right\rangle}=0 .
$$

Thus, for the special form of second moments in Eq. (21), time dependent part of $\epsilon_{i}$ does not contribute to $\overline{\left\langle\epsilon_{i}(t)\right\rangle}$ and we have

$$
\overline{\left\langle\epsilon_{i}(t)\right\rangle}=\hbar \omega \overline{\left\langle n_{i}(t)\right\rangle} \text {. }
$$

In order to arrive at equations of motion for $\overline{\left\langle\epsilon_{i}(t)\right\rangle}$ and $\overline{\left\langle n_{i}(t)\right\rangle}$, we differentiate both sides of Eq. (26) with respect to time and obtain

$$
\begin{aligned}
\frac{\partial \overline{\left\langle n_{i}(t)\right\rangle}}{\partial t} & =-\operatorname{Tr}\left(n_{i}\left(i \mathcal{L}_{0}+\mathcal{M}\right) e^{-i \mathcal{L}_{0} t-\mathcal{M} t} \rho(0)\right) \\
& =-\operatorname{Tr}\left(\overline{\rho(t)} \mathcal{M} n_{i}\right)
\end{aligned}
$$

To obtain the last expression we used explicit form of superoperators $\mathcal{L}_{0}$ and $\mathcal{M}$, cyclic permutations under trace and the fact that $\mathcal{L}_{0} n_{i}=0$. Using the explicit form of superoperator $\mathcal{M}$ we obtain the following equations for $\overline{\left\langle n_{i}(t)\right\rangle}$ and $\overline{\left\langle\epsilon_{i}(t)\right\rangle}$

$$
\begin{aligned}
& \frac{\partial \overline{\left\langle n_{i}(t)\right\rangle}}{\partial t}=\alpha^{2} \Delta_{i} \overline{\left\langle n_{i}(t)\right\rangle} \\
& \frac{\partial \overline{\left\langle\epsilon_{i}(t)\right\rangle}}{\partial t}=\alpha^{2} \Delta_{i} \overline{\left\langle\epsilon_{i}(t)\right\rangle}
\end{aligned}
$$

here $\alpha^{2}=\alpha_{1}^{2}+\alpha_{2}^{2}$ and $\Delta_{i}$ is a discrete Laplacian whose action on some function $f_{i}$ defined over discrete set of points $i$ is given by

$$
\Delta_{i} f_{i}=-2 f_{i}+f_{i+1}+f_{i-1} .
$$

In the case of cyclic boundary conditions Eqs. 31 and 32 are valid for all $i$ 's, whereas for open end boundaries they are valid for all $i$ 's except $i=1$ and $i=N$ for which we have

$$
\frac{\partial \overline{\left\langle n_{1}(t)\right\rangle}}{\partial t}=\alpha^{2}\left(\overline{\left\langle n_{2}(t)\right\rangle}-\overline{\left\langle n_{1}(t)\right\rangle}\right)
$$




$$
\begin{aligned}
\frac{\partial \overline{\left\langle n_{N}(t)\right\rangle}}{\partial t} & =\alpha^{2}\left(\overline{\left\langle n_{N-1}(t)\right\rangle}-\overline{\left\langle n_{N}(t)\right\rangle}\right), \\
\frac{\partial \overline{\left\langle\epsilon_{1}(t)\right\rangle}}{\partial t} & =\alpha^{2}\left(\overline{\left\langle\epsilon_{2}(t)\right\rangle}-\overline{\left\langle\epsilon_{1}(t)\right\rangle}\right), \\
\frac{\partial \overline{\left\langle\epsilon_{N}(t)\right\rangle}}{\partial t} & =\alpha^{2}\left(\overline{\left\langle\epsilon_{N-1}(t)\right\rangle}-\overline{\left\langle\epsilon_{N}(t)\right\rangle}\right)
\end{aligned}
$$

$\Delta_{i}$ is the discrete analogue of the continuous operator $l^{2} \partial^{2} / \partial x^{2}$ where $l$ is interatomic distance. We can see that for cyclic boundary conditions, $\overline{\left\langle n_{i}(t)\right\rangle}$ and $\overline{\left\langle\epsilon_{i}(t)\right\rangle}$ satisfy discrete versions of the diffusion and heat equations, respectively. For open end boundary conditions these equations are satisfied everywhere except the boundaries. In both cases the stationary solutions correspond to constant $\overline{\left\langle n_{i}(t)\right\rangle}$ and $\overline{\left\langle\epsilon_{i}(t)\right\rangle}$. Coefficients of diffusion $K_{d}$ and energy density diffusion $K_{h}$ are given by

$$
K_{d}=K_{h}=\alpha^{2} l^{2}
$$

In the case of diffusion coefficient $K_{d}$, Eq. 35 agrees with the earlier results [23, 24]. Time dependent diffusion coefficient obtained in Ref. 24] reduces to our result Eq. (35) for the special case of Hamiltonian (20) because all time dependent terms vanish for Hamiltonians with zero averaged hopping coefficient. We also note here that the closed equations for $\overline{\left\langle n_{i}(t)\right\rangle}$ and $\overline{\left\langle\epsilon_{i}(t)\right\rangle}$ are due to the simple form of Hamiltonian (20) and the resulting structure of superoperator $\mathcal{M}$. For more general Hamiltonians, such as those considered in Refs. [23, 24], closed equations exist only for the noise-averaged single particle density matrix, $\overline{\left\langle a_{i}^{\dagger} a_{j}\right\rangle}$. However, such more general models do not conserve noise averaged energy of the system and therefore cannot be used for studies of heat conductivity which is the primary focus of this paper. Including on-site noise terms in Hamiltonian (20) does conserve the noise averaged energy. However, inclusion of these terms does not affect the transport equations given by Eqs. 31, 32.

\section{COUPLING TO THE RESERVOIRS}

We would like to verify if a linear energy density distribution will be achieved if the lattice is placed between two stochastic Langevin-type reservoirs. To model coupling of the lattice to such reservoirs we will use our original open-end lattice model and modify the equations of motions for the operators for the first and last sites. These modifications are achieved by, firstly, including additional time-dependent noise terms of the form $\hbar \eta_{1}(t)\left(a_{1}^{\dagger}+a_{1}\right)$ and $\hbar \eta_{N}(t)\left(a_{N}^{\dagger}+a_{N}\right)$ for the first and last sites to Hamiltonian (20) and, secondly, by introducing a non-Hamiltonian damping for the creation and annihilation operators at the terminal sites with decay rates of, respectively, $\gamma_{1}$ and $\gamma_{N}$. The noise sources $\eta_{1}(t)$ and $\eta_{N}(t)$ are assumed to be not correlated to other noises in the Hamiltonian (20) or to each other and hence can be treated on the same basis as the hopping integral noises. We assume that

$$
\overline{\eta_{1}(t) \eta_{1}\left(t^{\prime}\right)}=\xi_{1}^{2} \delta\left(t-t^{\prime}\right), \overline{\eta_{N}(t) \eta_{N}\left(t^{\prime}\right)}=\xi_{N}^{2} \delta\left(t-t^{\prime}\right)
$$

Note that the stochastic coupling that we consider corresponds to reservoirs that exchange both energy and particles with the lattice. In the case of bosons, modified equations for energy density of the first and last sites are given by

$$
\begin{aligned}
\frac{\partial \overline{\left\langle\epsilon_{1}\right\rangle}}{\partial t} & =-\left(2 \gamma_{1}+\alpha^{2}\right) \overline{\left\langle\epsilon_{1}\right\rangle}+\alpha^{2} \overline{\left\langle\epsilon_{2}\right\rangle}+\omega \xi_{1}^{2} \\
\frac{\partial \overline{\left\langle\epsilon_{N}\right\rangle}}{\partial t} & =-\left(2 \gamma_{N}+\alpha^{2}\right) \overline{\left\langle\epsilon_{N}\right\rangle}+\alpha^{2} \overline{\left\langle\epsilon_{N-1}\right\rangle}+\omega \xi_{N}^{2}
\end{aligned}
$$

Equations of motion for energy densities at other sites remain unchanged and are given in Eq. (32). The stationary solutions of equations correspond to a linear distribution of $\overline{\left\langle\epsilon_{i}\right\rangle}$. The energy gradient $\theta$

$$
\theta=\frac{\overline{\left\langle\epsilon_{N}\right\rangle}-\overline{\left\langle\epsilon_{1}\right\rangle}}{N-1}
$$

is given in terms of reservoir coupling parameters by

$$
\theta=\frac{\omega\left(\gamma_{1} \xi_{N}^{2}-\gamma_{2} \xi_{N}^{2}\right)}{2(N-1) \gamma_{1} \gamma_{N}+\alpha^{2}\left(\gamma_{1}+\gamma_{N}\right)}
$$

while for the stationary state

$$
\overline{\left\langle\epsilon_{1}\right\rangle}=\frac{\omega \xi_{1}^{2}+\alpha^{2} \theta}{2 \gamma_{1}}, \quad \overline{\left\langle\epsilon_{N}\right\rangle}=\frac{\omega \xi_{N}^{2}-\alpha^{2} \theta}{2 \gamma_{1}}
$$

In the case of fermionic or spin operators, equations of motion for $\overline{\left\langle\epsilon_{1}\right\rangle}$ and $\overline{\left\langle\epsilon_{N}\right\rangle}$ are

$$
\begin{aligned}
\frac{\partial \overline{\left\langle\epsilon_{1}\right\rangle}}{\partial t}=-\left(2 \gamma_{1}+\alpha^{2}+2 \omega \xi_{1}^{2}\right) \overline{\left\langle\epsilon_{1}\right\rangle}+\alpha^{2} \overline{\left\langle\epsilon_{2}\right\rangle}+\omega \xi_{1}^{2} \\
\frac{\partial \overline{\left\langle\epsilon_{N}\right\rangle}}{\partial t}=-\left(2 \gamma_{N}+\alpha^{2}+2 \omega \xi_{N}^{2}\right) \overline{\left\langle\epsilon_{N}\right\rangle}+\alpha^{2} \overline{\left\langle\epsilon_{N-1}\right\rangle}+\omega \xi_{N}^{2} .
\end{aligned}
$$

Again, the stationary state corresponds to the linear distribution of $\overline{\left\langle\epsilon_{i}\right\rangle}$ with the energy density gradient given by

$$
\theta=\frac{\omega\left(\gamma_{1} \xi_{N}^{2}-\gamma_{N} \xi_{N}^{2}\right)}{2(N-1)\left(\gamma_{1}-\omega \xi_{1}^{2}\right)\left(-\gamma_{N}+\omega \xi_{N}^{2}\right)+\alpha^{2}\left(-\gamma_{1}-\gamma_{N}+\omega \xi_{1}^{2}+\omega \xi_{2}^{2}\right)}
$$


and

$$
\overline{\left\langle\epsilon_{1}\right\rangle}=\frac{\omega \xi_{1}^{2}+\alpha^{2} \theta}{2\left(\gamma_{1}+\omega \xi_{1}^{2}\right)}, \quad \overline{\left\langle\epsilon_{N}\right\rangle}=\frac{\omega \xi_{N}^{2}-\alpha^{2} \theta}{2\left(\gamma_{N}+\omega \xi_{N}^{2}\right)}
$$

Since in this case we are dealing with non-equlilibrium situation there is no unique way of introducing temperature in this case. However, we can say that the local temperature is proportional to the average energy density at a given site. Hence, we recover the macroscopic heat equation starting from a microscopic quantum description.

\section{DISCUSSION}

In this paper, we developed a quantum mechanical model for energy and particle transport in a linear chain with stochastic hopping coefficients. We present a set of specific conditions in which the noise-averaged energy expectation value will remain constant. Under such conditions we arrive at a quantum mechanical law for heat transport. Furthermore, we consider the case in which the chain can exchange both particles and energy with reservoirs on either end. Both the energy density gradient and particle density gradient can be obtained in closed form. We consider only gaussian noise for our stochastic coefficients. More detailed analysis will be required to develop a complete understanding of how well the averages represent the behavior of individual members of the ensemble. To reconfirm our theoretical results we performed a series of numerical simulations for our model (20) with a particle placed at one of the sites at $t=0$. As expected normal diffusion was observed for noise averaged time evolution for wide range of noise strengths. The simplicity of this model allows us to develop a more general treatment of quantum transport in a wide range of physical systems ranging from resonant electronic energy transport in DNA chains and organic glasses to Joule heating in molecular wires.

\section{Acknowledgments}

This work was funded in part through grants from the National Science Foundation and the Robert A. Welch foundation.
[1] A. Buldum, S. Ciraci, and C. Y. Fong, J. Phys.: Cond. Matter 12, 2249 (2000).

[2] K. Saito, Europhys. Lett. 61, 34 (2003).

[3] A. M. Macêdo, Phys. Rev. B 69, 155309 (2004).

[4] A. R. Kolovsky and H. J. Korsch, Phys. Rev. E 68, 046202 (2003).

[5] D. K. Wójcik and J. R. Dorfman, Phys. Rev. Lett. 90, 230602 (2003).

[6] K. Richter and M. Sieber, Physical Review Letters 89, 206801 (2002).

[7] K. Schwab, E. A. Henriksen, J. M. Worlock, and M. L. Roukes, Nature 404, 974 (2000).

[8] V. Pouthier and C. Girardet, Phys. Rev. B 66, 115322 (2002).

[9] F. Bonetto, J. L. Lebowitz, and L. Rey-Bellet, in Mathematical Physics 2000, edited by A. Fokas, A. Grigoryan, T. Kibble, and B. Zegarlinski (Imperial College Press, London, 2000), pp. 128-150.

[10] S. Lepri, R. Livi, and A. Politi, Phys. Rep. 377, 1 (2003).

[11] E. Fermi, J. Pasta, and S. Ulam, in Nonlinear Wave Motion (Am. Math. Soc, Providence, RI, 1974).

[12] R. Peierls, Ann. Phys. 3, 1055 (1929).

[13] S. Lepri, Phys. Rev. E 58, 7165 (1998).

[14] O. Narayan and S. Ramaswamy, Phys. Rev. Lett. 89, 200601 (2002).

[15] B. Li and J. Wang, Phys. Rev. Lett. 91, 044301 (2003).

[16] A. Pereverzev, Phys. Rev. E 68, 056124 (2003).
[17] B. Li, L. Wang, and B. Hu, Phys. Rev. Lett 88, 223901 (2002).

[18] B. Li, H. Zhao, and B. Hu, Phys. Rev. Lett 86, 63 (2001).

[19] B. Hu, B. Li, and H. Zhao, Phys. Rev. E 61, 3828 (2000).

[20] S. Lepri, R. Livi, and A. Politi, Phys. Rev. E 68, 067102 (2003).

[21] M. Bolsterli, M. Rich, and W. M. Visscher, Phys. Rev. A 1, 1086 (1970).

[22] F. Bonetto, J. L. Lebowitz, and J. Lukkarinen, J. Stat. Phys. 116, 783 (2004).

[23] A. Ovchinnikov and N. Erikhman, Sov. Phys. -JETP 40, 733 (1975).

[24] A. Madhukar and W. Post, Phys. Rev. Lett. 39, 1424 (1977).

[25] A. M. Jayannavar and N. Kumar, Phys. Rev. Lett. 48, 553 (1982).

[26] S. M. Girvin and G. D. Mahan, Phys. Rev. B 20, 4896 (1979).

[27] W. Fischer, H. Leschke, and P. Müller, Phys. Rev. Lett. 73, 1578 (1994).

[28] G. Lindblad, Commun. Math. Phys. 48, 119 (1976).

[29] D. Saldago and J. L. Sanchez-Gomez, Journal of Modern Optics 50, 975 (2003).

[30] H. Haug and A.-P. Jauho, Quantum Kinetics in Transport and Optics of Semiconductors (Springer, Berlin, 1996). 